\title{
Calculation and Validation of a Grain Boundary Complexion Diagram for Bi-doped Ni
}

\author{
Naixie Zhou ${ }^{a}$, Zhiyang Yu ${ }^{b, c}$, Yuanyao Zhang ${ }^{a}$, Martin P. Harmer ${ }^{b}$, Jian Luo ${ }^{a,}$ * \\ ${ }^{a}$ Department of NanoEngineering; Program of Materials Science and Engineering, University of California, \\ San Diego, La Jolla, CA 92093, U.S.A. \\ ${ }^{b}$ Department of Materials Science and Engineering, Lehigh University, Bethlehem, PA 18015, U.S.A. \\ 'School of Materials Science and Engineering, Xiamen University of Technology, Xiamen 361024, P. R. \\ China
}

\begin{abstract}
:
A grain boundary (GB) "phase" (complexion) diagram is computed via a lattice type statistical thermodynamic model for the average general GBs in Bi-doped Ni. The predictions are calibrated with previously-reported density functional theory calculations and further validated by experiments, including both new and old aberration-corrected scanning transmission electron microscopy characterization results as well as prior Auger electron spectroscopy measurements. This work supports a major scientific goal of developing GB complexion diagrams as an extension to bulk phase diagrams and a useful materials science tool.
\end{abstract}

Keywords: CALPHAD; complexion; grain boundary; interfacial thermodynamics; segregation

\footnotetext{
${ }^{*}$ Correspondence should be addressed to J.L. (jluo@alum.mit.edu)
} 


\section{Graphical Abstract}

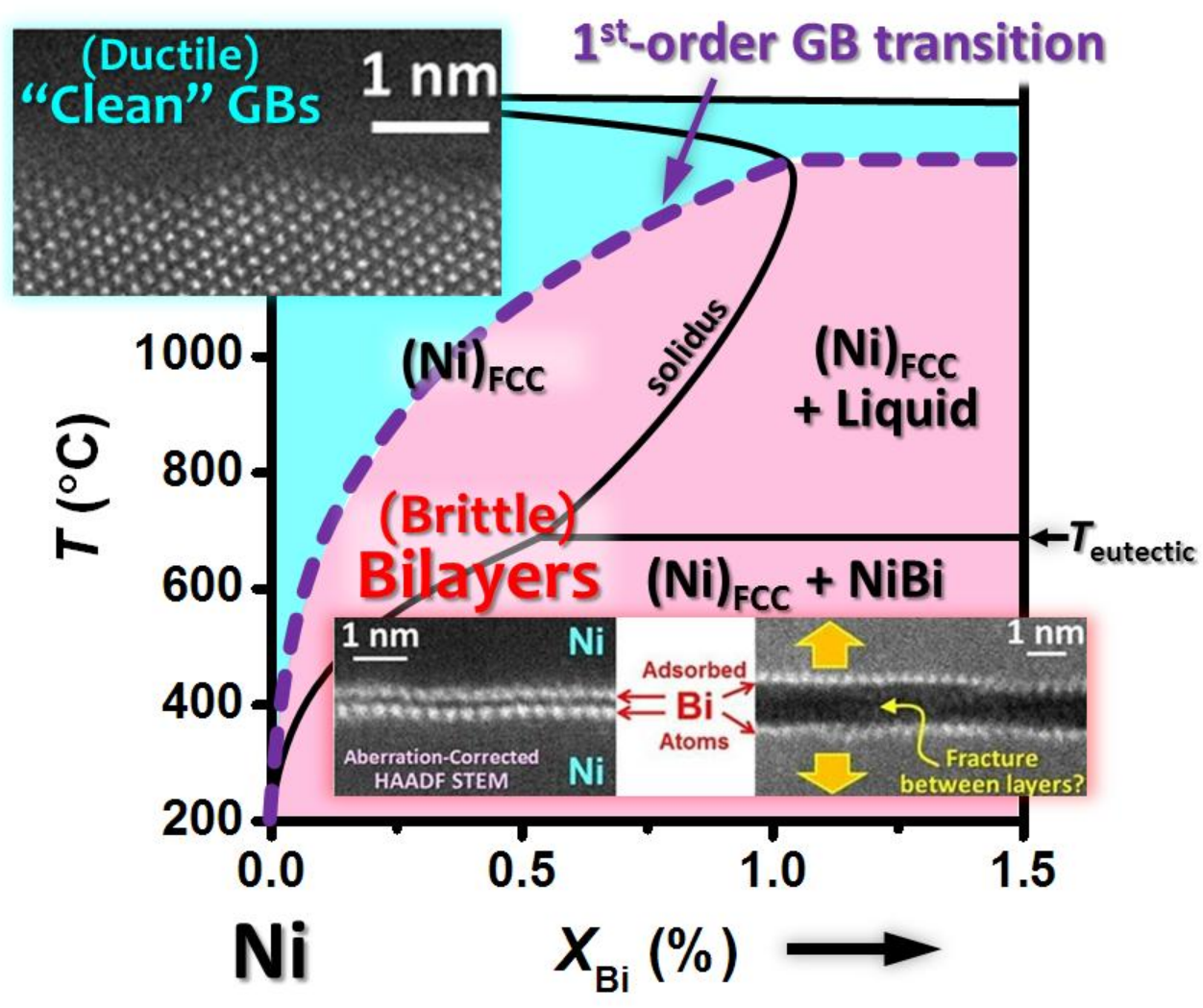


Grain boundaries (GBs) can undergo phase-like transformations, for example, premelting [1] or adsorption [2] transitions, which can influence a broad range of materials properties [3-5]. Thus, GBs can be treated as "interfacial phases" that are thermodynamically two-dimensional (2-D) despite that they have thermodynamically-determined interfacial widths as well as through-thickness compositional and structural gradients. A new term "complexion" was introduced to differentiate such 2-D interfacial phases from the conventional bulk phases defined by Gibbs $[1,3,4,6,7]$.

Phase diagrams are one of the most useful tools for materials engineering. Materials scientists have long recognized that phase-like behaviors at GBs can often control the fabrication processing, microstructural evolution, and materials properties [3, 6-12]. Thus, a potentially-transformative research is represented by the development of GB "phase" (a.k.a. complexion) diagrams as an extension to bulk phase diagrams and a generally-useful materials science tool. To support this goal, recent studies [9, 10, 13-16] have extended bulk CALPHAD (CALculation of PHAse Diagrams) methods to model coupled GB premelting and prewetting (a.k.a. the formation of nanoscale, impurity-based, quasi-liquid complexions) and subsequently constructed a class of "GB $\lambda$ diagrams" to represent the thermodynamic tendency for general GBs to disorder at high temperatures. Although the predicted trends have been validated with direct high-resolution transmission electron microscopy (HRTEM) [9, 14-19] and proven useful for forecasting sintering behaviors $[9,10,13-15,20]$, these GB $\lambda$ diagrams are not yet rigorous GB complexion diagrams with well-defined transition lines. An early report in 1999 [21] also constructed a GB complexion diagram for $\mathrm{Cu}-\mathrm{Bi}$ via a rather simple model that considered GBs as "quasi-liquid layers" to explain the GB segregation behaviors measured by Auger electron spectroscopy (AES), but more recent aberration-corrected scanning transmission electron microscopy (AC STEM) observed an ordered bilayer complexion in $\mathrm{Cu}-\mathrm{Bi}$ instead [22]. GB complexion diagrams with first-order transition lines and critical points have been constructed using diffuse-interface (phase-field) $[1,23,24]$ and lattice-type [16, 25-28] models, which have not yet been validated with experiments systematically, particularly by direct HRTEM or STEM characterization.

In this study, we computed a GB complexion diagram for average general GBs in $\mathrm{Bi}$-doped $\mathrm{Ni}$, for which a recent experimental study found ubiquitous formation of a bilayer complexion at virtually all general GBs at $700^{\circ} \mathrm{C}$ and $1100^{\circ} \mathrm{C}$ [29]. Furthermore, we calculated the GB transition lines for two special GBs based on the $0 \mathrm{~K}$ density functional theory (DFT) calculations reported in two prior studies $[30,31]$ for assessing the lattice model as well as representing (at least some) GB-to-GB variations. A computed GB complexion diagram has been constructed for the average general GBs in Bi-doped Ni and subsequently validated by both direct AC STEM characterization (including new AC STEM results for a 
specimen quenched from $1400^{\circ} \mathrm{C}$ collected in this study, along with prior results for $700^{\circ} \mathrm{C}$ and $1100^{\circ} \mathrm{C}$ [29]) and previously-reported AES analysis of GB segregation [32].

The GB energy of a binary A-B alloy can be expressed as:

$$
\gamma_{G B}=U_{G B}^{X S}-T S_{G B}^{X S}-\sum_{i}\left(\Gamma^{A} \mu^{A}+\Gamma^{B} \mu^{B}\right),
$$

where $U^{x \mathrm{~s}}$ is the excess internal energy, $S^{\mathrm{xs}}$ are the excess entropy, $\mu$ 's are bulk chemical potentials, and $\Gamma$ 's are the corresponding GB excesses (adsorption amounts). Similar to the free-energy functions of bulk phases, different functions of $\gamma_{\mathrm{GB}}$ can be written for different GB complexions and their intersections define GB transitions, e.g., the occurrence of a premelting transition from one complexion with low $S^{\mathrm{xs}}$ to another complexion with high $S^{\mathrm{xs}}$ with increasing temperature or an adsorption transition from one complexion with low $\Gamma$ to another complexion with high $\Gamma$ with increasing chemical potential difference. In this study, we focus on modeling GB adsorption (vs. prior studies [9, 10, 13-15] that forecasted interfacial disordering).

Here, we adopt a Wynblatt-Chatain multilayer adsorption model [33] to formulate an expression of $\gamma_{\mathrm{GB}}$ using an Ising type lattice model (assuming that each lattice point is occupied by either $\mathrm{Ni}$ or $\mathrm{Bi}$ atoms). The following expression is derived for a "general" (100) twist GB (to represent the average general $\Sigma \infty$ GBs, for which the fraction of "broken bonds" is set to ensure that $\gamma_{G B}^{(0)} / \gamma_{\text {surface }}^{(0)}=1 / 3$ to match the average value for "random" GBs in pure metals) to represent the average general GBs in Bidoped Ni:

$\gamma_{G B}=$ $2 \cdot \min \left\{0.5(1-P) Z_{v} N\left[2 X_{G B}^{1}\left(1-X_{G B}^{1}\right) \omega+X_{G B}^{1} e_{B i B i}+\left(1-X_{G B}^{1}\right) e_{N i N i}\right]-N \sum_{i=1}^{+\infty}\left[X_{G B}^{i} \Delta E_{e l s}^{i}\right]+N \omega \sum_{i=1}^{+\infty}\left[-z\left(X_{G B}^{i}-\right.\right.\right.$ $\left.\left.\left.X_{B u l k}\right)^{2}+z_{v}\left(X_{G B}^{i}-X_{G B}^{i+1}\right)^{2}\right]+N k T \sum_{i=1}^{+\infty}\left[X_{G B}^{i} \ln \left(\frac{X_{G B}^{i}}{X_{B u l k}}\right)+\left(1-X_{G B}^{i}\right) \ln \left(\frac{1-X_{G B}^{i}}{1-X_{B u l k}}\right)\right]\right\}$,

where $X_{G B}^{i}$ is the fraction of $\mathrm{Bi}$ atoms in the $i$-th layer near the GB core, $e_{\mathrm{Ni}-\mathrm{Ni}}(=-0.73 \mathrm{eV} / \mathrm{bond})$ and $e_{\mathrm{Bi}-\mathrm{Bi}}$ $(=-0.38 \mathrm{eV} /$ bond $)$ are bonding energies estimated from atomization enthalpies, $\omega\left[=e_{N i-B i}-\right.$ $\left.0.5\left(e_{N i-N i}+e_{B i-B i}\right)\right]$ is the regular-solution parameter (estimated to be $-0.017+1.1 \times 10^{-5} T \mathrm{eV} / \mathrm{bond}$ for Ni-Bi from the CALPHAD data [34]), $z(=12)$ is the total coordination number in FCC, $z_{\mathrm{v}}(=4)$ is the number of bonds per atom to one adjacent (100) plane (or $z_{\mathrm{v}}=3$ for twist (111) GBs), $P$ is the fraction of reconnected bonds that is set as $5 / 6$ to let $\gamma_{G B}^{(0)} / \gamma_{\text {surface }}^{(0)}=1 / 3$ to represent an average general $\mathrm{GB}$, and $N=$ $2.5 \times 10^{-5} \mathrm{~mole} / \mathrm{m}^{2}$ is the number of the lattice sites per unit area. $\Delta E_{\text {els }}^{i}$ is the elastic energy in the $i$-th layer, which decreases exponentially with the distance to the GB core $\left(h^{i}\right)$ according to:

$\Delta E_{\text {els }}^{i}=\Delta E_{\text {els }}^{1} \exp \left[-1.01\left(h^{i} / r_{B i}\right)^{1.53}\right]$, where $r_{\mathrm{Bi}}$ is the atomic radius of $\mathrm{Bi}$ and $\Delta E_{\text {els }}^{1} \quad(=0.27 \mathrm{eV} /$ atom for a $\mathrm{Bi}$ atom on the FCC Ni lattice) is obtained from the Friedel model [33]. The equilibrium 
compositional profiles are obtained by minimizing Eq. (2) $\left(\partial \gamma_{G B} / \partial X_{G B}^{i}=0\right)$, leading to a McLean type adsorption equation for each layer:

$$
\frac{X_{G B}^{i}}{1-X_{G B}^{i}}=\frac{X_{B u l k}}{1-X_{B u l k}} \exp \left(-\frac{\Delta H_{s e g}^{i}}{R T}\right)
$$

where $\Delta H_{\text {seg }}^{\mathrm{i}}$ is the adsorption enthalpy in the $i$-th layer and its expression can be found in Ref. [33]. For a set of given bulk composition (chemical potentials $\mu$ 's) and temperature $(T)$, the equilibrium GB compositional profile and GB energy can be obtained from Eq. (2) and Eq. (3) by minimizing $\gamma_{\mathrm{GB}}$ numerically via an iteration method. Then, the GB excess of solute can be calculated via: $\Gamma=$ $2 N \sum_{i}\left(X_{G B}^{i}-X_{C}\right)$.

Multiple complexions with different compositional profiles can satisfy Eq. (3) for a give set of $\mu$ 's and $T$ (see Fig. 1 for examples); in such a case, the compositional profile that produces the minimum $\gamma_{\mathrm{GB}}$ represents the equilibrium complexion and others are metastable. If two complexions have the same minimum $\gamma_{\mathrm{GB}}$, a GB transition can occur between them and it is first-order if first derivatives of $\gamma_{\mathrm{GB}}$ are discontinuous at the intersection.

Fig. 1 displays computed normalized GB energy $\left(\gamma_{\mathrm{GB}} / \gamma_{\mathrm{GB}}{ }^{(0)}\right)$ and adsorption $(\Gamma)$ vs. bulk composition $\left(X_{\mathrm{Bulk}}\right)$ curves for Bi-doped $\mathrm{Ni}$ at three different temperatures. Here, $\gamma_{\mathrm{GB}}{ }^{(0)}$ is computed to be $\sim 1.26 \mathrm{~J} / \mathrm{m}^{2}$ for the average general GBs in pure $\mathrm{Ni}$, which is consistent with experiments $\left(\sim 1 \mathrm{~J} / \mathrm{m}^{2}\right)$ and DFT calculations $\left(1.2-1.4 \mathrm{~J} / \mathrm{m}^{2}\right)[30,35,36]$. At each temperature, the GB undergoes a first-order phase-like (complexion) transformation with increasing bulk composition, from a "clean" GB to a bilayer; here, both the "clean" GB and the bilayer are nominal as the actual GB excess $(\Gamma)$, which varies with the chemical potential, is around the nominal value of 0 or 2 monolayer(s) (Fig. 1). These GB transitions are first-order, as indicated by the discontinuities in the slopes in the $\gamma_{\mathrm{GB}} / \gamma_{\mathrm{GB}}{ }^{(0)} v s . X_{\mathrm{Bulk}}$ curves and finite jumps in the $\Gamma$ vs. $X_{\text {Bulk }}$ curves. The first-order GB transition also occur at $1400^{\circ} \mathrm{C}$, but above the solidus line, so that the bilayer is not an equilibrium complexion. It is interesting to further note that the metastable GB transition is nearly continuous at $1400^{\circ} \mathrm{C}$ (see Fig. 1(f)); thus, a metastable critical point may exist above $1400^{\circ} \mathrm{C}$ in the supersaturated region. Likewise, high-order complexions may exist in the supersaturated region (beyond the bulk solid solubility limits). In general, several other types of complexions $[1,3,4,6,7]$ may exist in other alloys with different thermodynamic parameters; in the current case, however, bilayers and "clean" GBs are the only two equilibrium GB complexions predicted from the lattice model for Ni-Bi, which is consistent with the experimental observations discussed subsequently. 
The lattice type models have several limitations. They do not consider possible structural changes at GBs (by placing all atoms on the lattice) and rely on empirical parameters. Thus, we further compare this model with two prior DFT calculations [30, 31] that consider the detailed atomic structures (for selected special GBs at $T=0 \mathrm{~K})$. Fig. 2 (a) and (b) compare the $\left(\gamma_{\mathrm{GB}}-\gamma_{\mathrm{GB}}{ }^{(0)}\right) v s$. chemical potential difference curves for two twist "general" GBs calculated using the lattice model and CALPHAD data with two independent DFT calculations [30,31] for two $\Sigma 5$ tilt and one $\Sigma 3$ twist GBs at $T=0 \mathrm{~K}$. There are several differences between the lattice model and DFT calculations. First, the relaxed bilayer structures obtained by DFT is less dense (with reconstructions) than that of the lattice model. Second, $\Delta \gamma_{G B}^{B L}$ at $\Delta \mu=0$ is higher for DFT results because of the lower $\Gamma$. Third, the Bi chemical potentials in the NiBi compound obtained from the CALPHAD and DFT are somewhat different (noting that the CALPHAD data were fitted referencing to the bulk compound so that the relative chemical potentials are likely more relevant). Finally, the lattice model is designed to represent the average general $(\Sigma \infty)$ GBs, whereas DFT calculations were conducted for special $\Sigma 5$ tilt GBs and for the lowest-energy $\Sigma 3$ (111) twist GB [29-31]. Nonetheless, it is interesting to note that the relative stabilities of the "clean" vs. bilayer complexions at the average general GBs represented the lattice model are between the two DFT calculations for $\Sigma 5$ (210) and $\Sigma 5$ (310) tilt GBs [30,31] (Fig. 2), which will be discussed further subsequently.

The differences in DFT calculations represents at least some of the anisotropy of GB complexion transitions. Calculations using the lattice model represent "average" general GBs. In fact, computed $\Delta \gamma v s$. $\Delta \mu$ curves are almost identical for two types of general twist GBs (Fig. 2(a)), both of which were selected to represent average general GBs, implying the robustness of this lattice model.

Furthermore, we used the DFT results (obtained for $T=0 \mathrm{~K}$ ) reported in two prior studies [30, 31] to estimate the complexion transition lines at finite temperatures using the following approximation: $X_{\text {clean }_{\rightarrow} \mathrm{BL}} \approx X_{\text {solvus }} \cdot \exp \left(-\Delta \mu_{\mathrm{BL}-\mathrm{NiB}} / \mathrm{R} T\right)$, where the $X_{\text {solvus }}$ is the solvus composition calculated by CALPHAD and $\Delta \mu_{\mathrm{BL}-\mathrm{NiBi}}$ is the chemical potential difference calculated by DFT at $T=0 \mathrm{~K}$; this equation assumes that the Ni-Bi forms a regular solution and $\Delta \mu_{\mathrm{BL}-\mathrm{NiBi}}$ is constant independent of temperature. We further plot the two GB transition lines estimated based on the two prior OK DFT calculations for $\Sigma 5$ (210) and $\Sigma 5$ (310) tilt GBs $[30,31]$ in the bulk Ni-Bi phase diagram, along with the GB transition line calculated from the lattice model for twist general GBs, in Fig. 2(c). These three GB complexion transition lines represent some GB-to-GB variations, as well as errors from different models and data; we further note that the bilayer complexion is completely unstable at the low-energy $\Sigma 3$ (111) twist GB (Fig. 2(b)), which represents an extreme case of anisotropy. Significant GB-to-GB variations in GB transitions, as partially illustrated in Fig. 2, are well expected. 
Nonetheless, all three calculated transition lines in Fig. 2(c) by different methods and/or for different GBs follow the same trends. The comparison with GB transition lines estimated from prior OK DFT calculations for two $\Sigma 5$ tilt GBs suggest the computed GB transition line for the average general GBs is reasonable. Thus, the lattice model predictions are consistent with/supported by DFT calculations.

Finally, we have constructed a GB complexion diagram for the average general GBs Bi-doped Ni in Fig. 3, which shows the stability regions for two complexions - a nominally "clean" complexion and a bilayer complexion (represented by two different colors in Fig. 3) - separated by the first-order GB transition line (the purple dashed line) computed from the lattice model. Our prior study has already demonstrated via AC STEM high-angle annular dark-field (HAADF) imaging that bilayers form ubiquitously at solid-liquid coexistence (along the solidus line) at $700^{\circ} \mathrm{C}$ and $1100^{\circ} \mathrm{C}$ [29]. In this study, the lattice model (Fig. 1 and Fig. 3) further suggested that bilayer should vanish at $T>\sim 1300^{\circ} \mathrm{C}$ along the solidus line for general GBs. To verify this prediction, we conducted an additional experiment to anneal a $\mathrm{Ni}$ polycrystalline specimen in direct contact with an equilibrium Bi-Ni liquid at $1400^{\circ} \mathrm{C}$ and subsequently quenched it to preserve its high-temperature GB configuration. The TEM specimens were prepared by a focused ion beam and characterized with AC STEM. Electron backscattering diffraction was used to ensure the selected GB is a general GB. Here, we adopted the same experimental procedure as that in the prior study [29], except for a specimen annealed and quenched at/from a higher temperature of $1400^{\circ} \mathrm{C}$. The HAADF STEM image, as shown in the top-right corner of Fig. 3, verified the prediction of the computed GB complexion diagram, i.e., the bilayer vanished.

The occurrence of a first-order GB transition from nominally clean GBs to nominal bilayers in the single phase region is also consistent with a prior AES study of GB segregation by Chang and Huang [32], who found that a first-order $\mathrm{GB}$ transition occurred at $\sim 400^{\circ} \mathrm{C}$ in $\mathrm{Ni}+90$ wt. ppm Bi (i.e., $X_{\mathrm{Bi}} \approx 0.0026 \%$ ) specimens, which falls almost exactly on the calculated transition line in the complexion diagram shown in Fig. 3, although the observed transition temperature was higher than that predicted in Fig. 3 for specimens with a higher Bi content (meaning that the slope of the GB transition line suggested by that AES study was higher) [32]. Overall, the agreements between the computed GB complexion diagram for the general GBs (Fig. 3) and various experiments (both STEM and AES) are satisfactory.

In summary, we have computed a complexion diagram for the average general GBs in Bi-doped $\mathrm{Ni}$ using a lattice model and CALPHAD data. The computed results are critically assessed with two earlier reports of OK DFT calculations. The computed GB complexion (phase) diagram has been systematically validated with experiments, including direct AC STEM characterization (both previously reported data for specimens quenched from $700^{\circ} \mathrm{C}$ and $1100^{\circ} \mathrm{C}$ [29] and a new experiment on a specimen quenched from $1400^{\circ} \mathrm{C}$ ) and an early AES study of GB segregation [32]. In addition to the earlier development GB 
$\lambda$-diagrams that can forecast the trends in GB disordering and related sintering behaviors at high temperatures $[9,14,15,17-19]$, this study constructed a more rigorous (yet simple) GB complexion diagram with well-defined transition lines that can be verified by experiments. Future research should be conducted to compute and validate other GB complexion diagrams to realize a potentially-transformative scientific goal of systematically constructing GB complexion diagrams as a new materials science tool.

We gratefully acknowledge the support from the Office of Naval Research (N00014-11-1-0678). N.Z. and J.L. also acknowledge partial support from a Vannevar Bush Faculty Fellowship (N00014-16-12569). We thank Denise Yin and Ken Wu for their assistance in preparing TEM specimens. 


\section{List of Figure Captions}

Figure 1. Calculated normalized GB energy $\left(\gamma_{\mathrm{GB}} / \gamma_{\mathrm{GB}}{ }^{(0)}\right) v$ s. bulk Bi composition $\left(\mathrm{X}_{\mathrm{Bi}}\right)$ curves for Bi-doped $\mathrm{Ni}$ at (a) $700^{\circ} \mathrm{C}$, (b) $1100^{\circ} \mathrm{C}$, and (c) $1400^{\circ} \mathrm{C}$, respectively, and the corresponding computed GB excesses of the solute ( $\Gamma^{\prime}$ s) at (d) $700^{\circ} \mathrm{C}$, (e) $1100^{\circ} \mathrm{C}$, and (f) $1400^{\circ} \mathrm{C}$, respectively. GB complexion transitions occur when $\gamma_{\mathrm{GB}} / \gamma_{\mathrm{GB}}{ }^{(0)} v$ v. $X_{\mathrm{Bi}}$ curves for the "bilayers" (red lines) and the "clean" GBs (blue lines) intersect. The first-order transitions correspond to the abrupt increases (finite jumps) of absorption in (d)(f) or the associated discontinuities in the slopes in (a)-(c). Dashed lines represent the metastable regions of the complexions. The dotted lines in (c) and (f) represent the supersaturated (metastable) region beyond the bulk solidus line (that would occur only if the formation of the liquid phase was inhibited). Noting that the metastable GB transition is nearly continuous at $1400^{\circ} \mathrm{C}$, as shown in (f), so that a metastable critical point may exist above $1400^{\circ} \mathrm{C}$.

Figure 2. Comparison of computed relative GB energies $\left(\Delta \gamma=\gamma_{\mathrm{GB}}{ }^{\text {bilayer }}-\gamma_{\mathrm{GB}}{ }^{(0)}\right)$ of the Bi-based bilayers at Ni GBs as functions of Bi chemical potential $\Delta \mu_{\mathrm{Bi}}$ at $T=0 \mathrm{~K}$ using (a) a Wynblatt-Chatain lattice model for two general twist GBs to represent the average general GBs and (b) DFT calculations for a $\Sigma 5(210)$ tilt GB and a $\Sigma 3(111)$ twist GB using data from Ref. [30] as well as a $\Sigma 5(310)$ tilt GB using data from Ref. [31]. (c) The corresponding first-order ("clean" to "bilayer") GB phase-like transition lines at finite temperatures, which were subsequently computed for the average general GBs using a lattice model based on CALPHAD data and for two special $\Sigma 5$ GBs using a simplified approach (described in the text) based on the prior DFT results $[30,31]$ shown in panel (b), are plotted as the purple dashed lines in the bulk NiBi bulk phase diagram (where the bulk composition, $X_{\mathrm{Bi}}$, is in a logarithmical scale). No GB transition occurs for the $\Sigma 3(111)$ twist GB, a low-energy GB where the bilayer does not form.

Figure 3. A computed GB complexion diagram, along with the corresponding STEM images that experimentally verified the prediction for three selected compositions on the solidus line labeled in the diagram. Black solid lines represent bulk phase boundaries and purple dashed line indicates the first-order GB transition line computed from a lattice model using CALPHAD data for the average general GBs in Bi-doped Ni. The observation of bilayers in Bi-saturated specimens at $700^{\circ} \mathrm{C}$ and $1100^{\circ} \mathrm{C}$ was reported in Ref. [29] and the STEM characterization of Bi-saturated specimens at $1400^{\circ} \mathrm{C}$ that confirmed the occurrence of GB transition is a new result of this study. 


\section{References:}

[1] M. Tang, W.C. Carter, R.M. Cannon, Phys. Rev. B 73 (2006) 024102.

[2] S. Ma, K. Meshinchi Asl, C. Tansarawiput, P.R. Cantwella, M. Qi, M.P. Harmer, J. Luo, Scripta Mater. 66 (2012) 203-206.

[3] P.R. Cantwell, M. Tang, S.J. Dillon, J. Luo, G.S. Rohrer, M.P. Harmer, Acta Mater. 62 (2014) 1-48.

[4] M.P. Harmer, Science 332 (2011) 182-183.

[5] J. Luo, Crit. Rev. Solid State Mater. Sci. 32 (2007) 67-109.

[6] S.J. Dillon, M. Tang, W.C. Carter, M.P. Harmer, Acta Mater. 55 (2007) 6208-6218

[7] W.D. Kaplan, D. Chatain, P. Wynblatt, W.C. Carter, J. Mater. Sci. 48 (2013) 5681-5717.

[8] J. Luo, J. Materiomics 1 (2015) 22-32.

[9] J. Luo, J. Am. Ceram. Soc. 95 (2012) 2358-2371.

[10] J. Luo, Curr. Opin. Solid State Mater. Sci. 12 (2008) 81-88.

[11] J. Luo, Crit. Rev. Solid State Mater. Sci. 32 (2007) 67-109.

[12] J. Luo, Corrosion 72 (2015) 897-910.

[13] J. Luo, X.M. Shi, Appl. Phys. Lett. 92 (2008 ) 101901

[14] X. Shi, J. Luo, Phys. Rev. B 84 (2011) 014105.

[15] N. Zhou, J. Luo, Acta Mater. 91 (2015) 202-216.

[16] N. Zhou, T. Hu, J. Luo, Curr. Opin. Solid State Mater. Sci. 20 (2016) 268-277.

[17] X. Shi, J. Luo, Appl. Phys. Lett. 94 (2009) 251908.

[18] V.K. Gupta, D.H. Yoon, H.M. Meyer III, J. Luo, Acta Mater. 55 (2007) 3131-3142.

[19] J. Luo, V.K. Gupta, D.H. Yoon, H.M. Meyer, Appl. Phys. Lett. 87 (2005) 231902.

[20] X. Shi, J. Luo, Phys. Rev. Lett. 105 (2010) 236102.

[21] L.S. Chang, E. Rabkin, B.B. Straumal, B. Baretzky, W. Gust, Acta Mater. 47 (1999) 4041-46.

[22] A. Kundu, K.M. Asl, J. Luo, M.P. Harmer, Scripta Mater. 68 (2013) 146-149.

[23] M. Tang, W.C. Carter, R.M. Cannon, Phys. Rev. Lett. 97 (2006) 075502.

[24] Y. Mishin, W.J. Boettinger, J.A. Warren, G.B. McFadden, Acta Mater. 57 (2009) 3771-3785.

[25] P. Wynblatt, D. Chatain, Mater. Sci. Eng. A 495 (2008) 119-125.

[26] J. Luo, Appl. Phys. Lett. 95 (2009) 071911.

[27] J.M. Rickman, H.M. Chan, M.P. Harmer, J. Luo, Surf. Sci. 618 (2013) 88-93.

[28] J.M. Rickman, J. Luo, Curr. Opin. Solid State Mater. Sci. 20 (2016) 225.

[29] J. Luo, H. Cheng, K.M. Asl, C.J. Kiely, M.P. Harmer, Science 333 (2011) 1730-1733.

[30] Q. Gao, M. Widom, Phys. Rev. B 90 (2014) 144102.

[31] J. Kang, G.C. Glatzmaier, S.H. Wei, Phys. Rev. Lett. 111 (2013) 055502.

[32] L.S. Chang, K.B. Huang, Scripta Mater. 51 (2004) 551-555.

[33] P. Wynblatt, D. Chatain, Metall. Mater. Trans. A 37A (2006) 2595-2620.

[34] G. Vassilev, V. Gandova, P. Docheva, Cryst. Res. Technol. 44 (2009) 25-30.

[35] T. Roth, Mater. Sci. Eng. 18 (1975) 183-192.

[36] Y. Masatake, S. Motoyuki, K. Hideo, J. Phys.: Condens. Matter 16 (2004) 3933. 

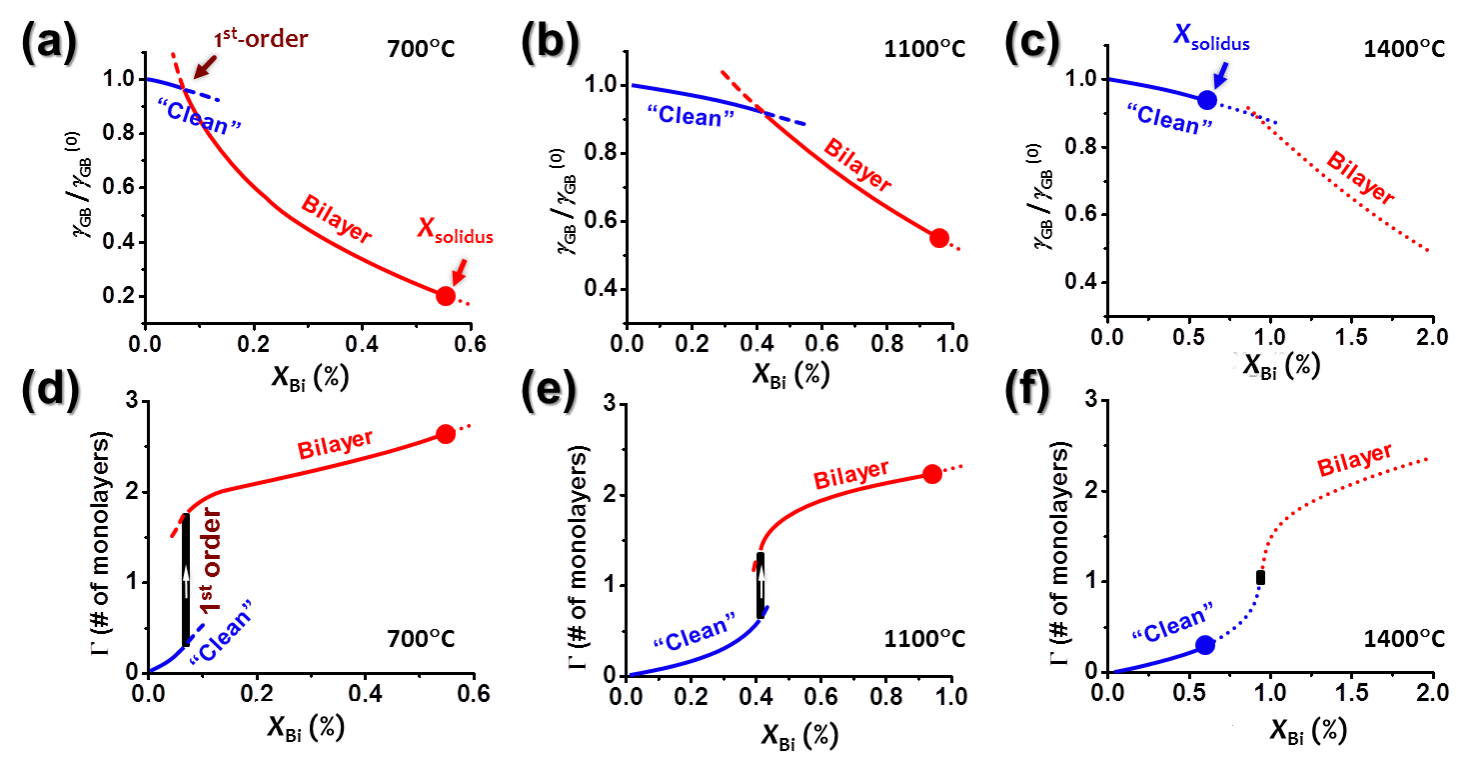

Figure 1. Calculated normalized GB energy $\left(\gamma_{\mathrm{GB}} / \gamma_{\mathrm{GB}}{ }^{(0)}\right) v$ s. bulk Bi composition $\left(\mathrm{X}_{\mathrm{Bi}}\right)$ curves for Bi-doped $\mathrm{Ni}$ at (a) $700^{\circ} \mathrm{C}$, (b) $1100^{\circ} \mathrm{C}$, and (c) $1400^{\circ} \mathrm{C}$, respectively, and the corresponding computed GB excesses of the solute $\left(\Gamma\right.$ 's) at (d) $700^{\circ} \mathrm{C}$, (e) $1100^{\circ} \mathrm{C}$, and (f) $1400^{\circ} \mathrm{C}$, respectively. GB complexion transitions occur when $\gamma_{\mathrm{GB}} / \gamma_{\mathrm{GB}}{ }^{(0)}$ vs. $X_{\mathrm{Bi}}$ curves for the "bilayers" (red lines) and the "clean" GBs (blue lines) intersect. The first-order transitions correspond to the abrupt increases (finite jumps) of absorption in (d)(f) or the associated discontinuities in the slopes in (a)-(c). Dashed lines represent the metastable regions of the complexions. The dotted lines in (c) and (f) represent the supersaturated (metastable) region beyond the bulk solidus line (that would occur only if the formation of the liquid phase was inhibited). Noting that the metastable GB transition is nearly continuous at $1400^{\circ} \mathrm{C}$, as shown in (f), so that a metastable critical point may exist above $1400^{\circ} \mathrm{C}$. 

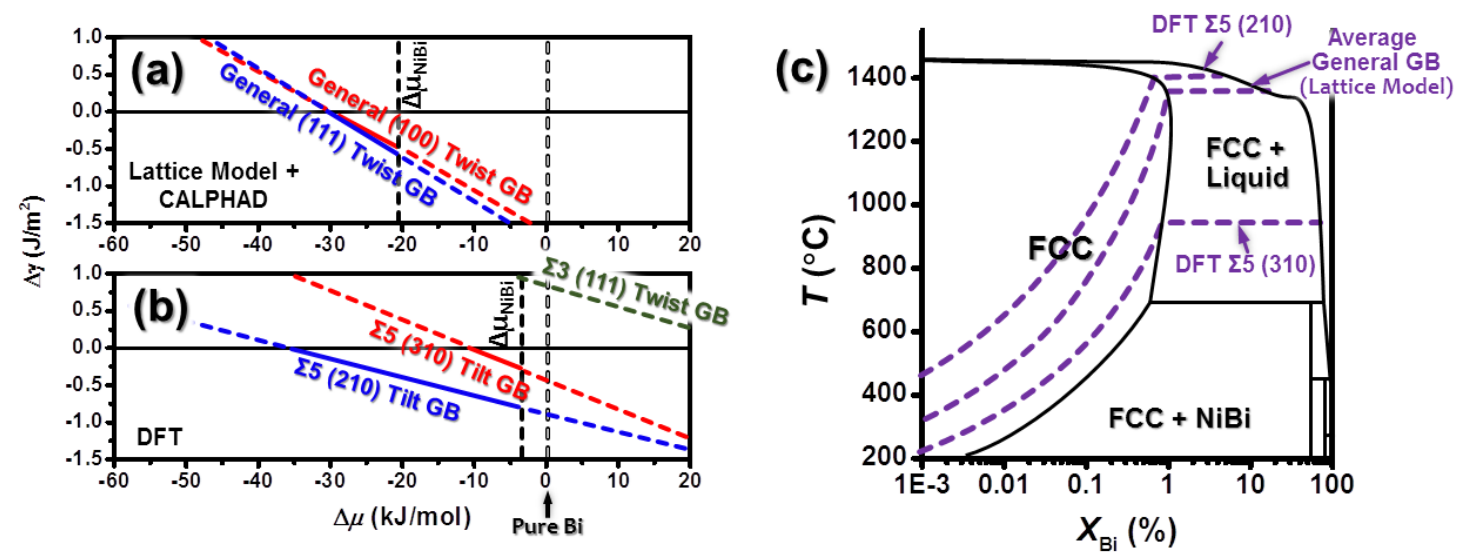

Figure 2. Comparison of computed relative GB energies $\left(\Delta \gamma=\gamma_{\mathrm{GB}}{ }^{\text {bilayer }}-\gamma_{\mathrm{GB}}{ }^{(0)}\right)$ of the Bi-based bilayers at Ni GBs as functions of Bi chemical potential $\Delta \mu_{\mathrm{Bi}}$ at $T=0 \mathrm{~K}$ using (a) a Wynblatt-Chatain lattice model for two general twist GBs to represent the average general GBs and (b) DFT calculations for a $\Sigma 5(210)$ tilt GB and a $\Sigma 3(111)$ twist GB using data from Ref. [30] as well as a $\Sigma 5(310)$ tilt GB using data from Ref. [31]. (c) The corresponding first-order ("clean" to "bilayer") GB phase-like transition lines at finite temperatures, which were subsequently computed for the average general GBs using a lattice model based on CALPHAD data and for two special $\Sigma 5 \mathrm{GBs}$ using a simplified approach (described in the text) based on the prior DFT results $[30,31]$ shown in panel (b), are plotted as the purple dashed lines in the bulk NiBi bulk phase diagram (where the bulk composition, $X_{\mathrm{Bi}}$, is in a logarithmical scale). No GB transition occurs for the $\Sigma 3(111)$ twist GB, a low-energy GB where the bilayer does not form. 


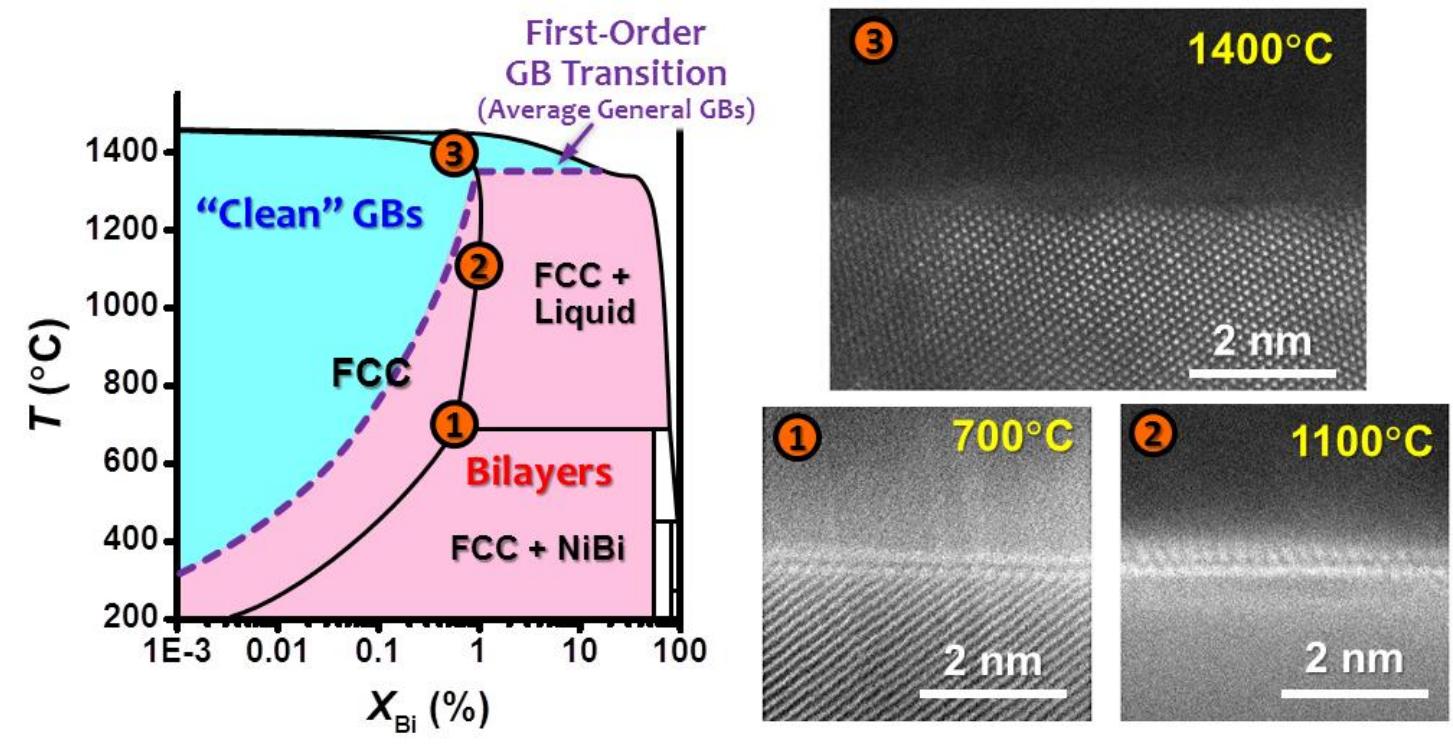

Figure 3. A computed GB complexion diagram, along with the corresponding STEM images that experimentally verified the prediction for three selected compositions on the solidus line labeled in the diagram. Black solid lines represent bulk phase boundaries and purple dashed line indicates the first-order GB transition line computed from a lattice model using CALPHAD data for the average general GBs in Bi-doped Ni. The observation of bilayers in Bi-saturated specimens at $700^{\circ} \mathrm{C}$ and $1100^{\circ} \mathrm{C}$ was reported in Ref. [29] and the STEM characterization of Bi-saturated specimens at $1400^{\circ} \mathrm{C}$ that confirmed the occurrence of GB transition is a new result of this study. 